\title{
Competition between human cells by entosis
}

Qiang Sun ${ }^{1,2}$, Tianzhi Luo ${ }^{3}$, Yixin Ren ${ }^{3}$, Oliver Florey ${ }^{4}$, Senji Shirasawa ${ }^{5}$, Takehiko Sasazuki ${ }^{6}$, Douglas N Robinson ${ }^{3}$, Michael Overholtzer ${ }^{2,7}$

${ }^{1}$ Laboratory of Cell Engineering, Institute of Biotechnology, Beijing 100071, China; ${ }^{2}$ Cell Biology Program, Memorial Sloan-Kettering Cancer Center, New York, NY 10065, USA; ${ }^{3}$ Department of Cell Biology, Johns Hopkins University School of Medicine, Baltimore, MD 21205, USA; ${ }^{4}$ Signalling Programme, The Babraham Institute, Cambridge, CB22 3AT, UK; ${ }^{5}$ Department of Cell Biology, School of Medicine, Fukuoka University, Fukuoka 814-0180, Japan; ${ }^{6}$ Department of Pathology, Research Institute, International Medical Center of Japan, Tokyo 163-8655, Japan; ${ }^{7}$ BCMB Allied Program, Weill Cornell Medical College, 1300 York Avenue, New York, NY 10065, USA

Human carcinomas are comprised of complex mixtures of tumor cells that are known to compete indirectly for nutrients and growth factors. Whether tumor cells could also compete directly, for example by elimination of rivals, is not known. Here we show that human cells can directly compete by a mechanism of engulfment called entosis. By entosis, cells are engulfed, or cannibalized while alive, and subsequently undergo cell death. We find that the identity of engulfing ("winner") and engulfed ("loser") cells is dictated by mechanical deformability controlled by RhoA and actomyosin, where tumor cells with high deformability preferentially engulf and outcompete neighboring cells with low deformability in heterogeneous populations. We further find that activated Kras and Rac signaling impart winner status to cells by downregulating contractile myosin, allowing for the internalization of neighboring cells that eventually undergo cell death. Finally, we compute the energy landscape of cell-in-cell formation, demonstrating that a mechanical differential between winner and loser cells is required for entosis to proceed. These data define a mechanism of competition in mammalian cells that occurs in human tumors.

Keywords: entosis; cell competition; cell cannibalism; cell-in-cell structure; tumor evolution; Kras; Rho GTPase Cell Research (2014) 24:1299-1310. doi:10.1038/cr.2014.138; published online 24 October 2014

\section{Introduction}

The cell number of each tissue in metazoan organisms is carefully controlled as disruptions in cell number homeostasis can lead to pathologies associated with inappropriate cell loss (e.g., tissue degeneration) or cell gain (e.g., tumor growth). Programs that limit clonal expansion within tissues, such as cell death or senescence, can be executed cell-autonomously, but evidence has emerged that individual cells within a tissue can also eliminate neighboring cells threatening tissue integrity by inducing cell death non-cell-autonomously. Examples of

Correspondence: Michael Overholtzer ${ }^{\mathrm{a}}$, Qiang Sun ${ }^{\mathrm{b}}$

${ }^{a}$ Tel: +1-212-639-6536; Fax: +1-212-794-4342

E-mail: overhom1@mskcc.org

${ }^{\mathrm{b}}$ Tel: +86-10-66948820

E-mail: sunqiang1975@126.com

Received 31 December 2013; revised 7 March 2014; accepted 30 July 2014; published online 24 October 2014 such "competition" between individual cells, where "fit" cells eliminate neighboring "less-fit" cells, have emerged from genetic studies in Drosophila melanogaster. In this system, wild-type cells, for example, outcompete less-fit cells harboring mono-allelic deletion of ribosomal protein genes ("Minute" cells) by inducing their death $[1,2]$. Similarly, cells overexpressing the dmyc oncogene, socalled "super-competitors", overpopulate at the expense of wild-type cells that are eliminated [3,4]. In each case, the total cell number, or overall tissue size, is maintained at the expense of "loser" cells that are killed by "winners", through a program termed "cell competition".

It has been argued that cell competition in Drosophila requires cell engulfment to induce the death of "loser" cells. For example, competition between wild-type and Minute cells requires engulfment at the borders of rival clones, mediated by the small GTPase Rac-1, to promote both the death and clearance of loser cells, suggesting that engulfment plays a direct role in allowing winner cells to kill losers [5]. Similarly, clones of cells with mu- 
tations in the tumor suppressor genes scrib and $d l g$ have been shown to be engulfed and eliminated by neighboring wild-type cells [6], suggesting that cell competition can be mediated in some contexts by engulfment-induced cell death that follows direct contact between winners and losers.

The identification of cell competition in Drosophila has led to the speculation that similar modes of competition between mammalian cells could occur in human tumors $[7,8]$. The observation that mammalian cells expressing activated Src or Kras are extruded when surrounded by normal cells in monolayer cultures has demonstrated one mode of competitive interactions between neighboring mammalian cells, which could influence tumorigenesis $[9,10]$. Similarly, knockdown of the polarity protein Scribble and the Lgl-binding protein Mahjong, whose loss induces cell competition in Drosophila, leads to death of mammalian cells only when knockdown cells are mixed with wild-type cells in culture $[11,12]$. While cell engulfment is argued to control cell competition in Drosophila, no examples of competition between mammalian cells where engulfment could contribute to the killing of loser cells by winners have been identified.

Recently, a mechanism of mammalian cell death called entosis was reported, which is induced by cell engulfment [13]. The cannibalistic cell-in-cell structures formed by this mechanism are found in a variety of human tumors [13-15], and roles for this process in both tumor suppression and promotion have been proposed [15-17]. Although entotic cells are engulfed while alive, the majority undergo cell death, and are in fact killed by the neighboring cells into which they are engulfed [17]. We thus hypothesized that this mechanism of cell cannibalism could allow neighboring tumor cells to undergo a form of competition, as "winner" cells could ingest and kill neighboring "loser" cells. Here we investigate cell properties and signaling mechanisms that define winner and loser cell status during entosis, and examine whether entosis can mediate cell competition between tumor cells.

\section{Results}

\section{Entosis mediates competition between human cells}

As shown in a companion paper by Sun et al. [18], sibling tumor cells can undergo entosis in suspension cultures, a process associated with the accumulation of actomyosin in only one cell of a pair, demonstrating that "winner" and "loser" cell status can be dictated by transient differences between isogenic cells. However, we wondered whether genetic differences between cells in heterogeneous populations could contribute to the determination of winner and loser status, and in this manner entosis could mediate competition. Interestingly, non-tumorigenic MCF10A mammary epithelial cells were nearly always engulfed, becoming losers, when they were mixed with breast tumor cells expressing endogenous (MCF7) or exogenous (MDA-MB-231-E-cadherin) E-cadherin (Figure 1A). Similarly, non-tumorigenic pancreatic cells (HPDE) were losers when mixed with pancreatic tumor cells (PancTu; Figure 1A). In general, tumor cells were winners over non-tumor cells even when mixed with cells of different cell types (Figure 1A). We noted that tumor cells used in our cell mixing assays had lower levels of total phosphorylated myosin light chain 2 (pMLC2) than both non-tumor cell lines, suggesting that a low setpoint of contractile myosin might correlate with winner status. (Supplementary information, Figure S1A). Between different tumor cell lines, winner and loser cell status varied; for example, MCF7 cells were winners when mixed with non-tumorigenic MCF10A and HPDE cells, but were losers when mixed with some tumor cells such as VmCUB3 cells (Figure 1B), which exhibit a lower level of pMLC2 (Supplementary information, Figure S1A). Once VmCUB3 winner cells engulfed MCF7 loser cells (Supplementary information, Figure S1B), MCF7 loser cells frequently underwent cell death when examined through time (Supplementary information, Figure S1C). Remarkably, the preferential engulfment of loser cells by winners and the induction of loser cell death were recapitulated in mixed xenograft tumors in vivo (Figure 1C-1E and Supplementary information, Figures S1D, S1E and S2). In this context, engulfment and entotic cell death were suppressed by treatment of mice with the ROCK inhibitor Y27632, consistent with the engulfment program entosis (Figure $1 \mathrm{C}$ and $1 \mathrm{E}$ ).

To examine whether cell engulfment can promote competition between tumor cell populations, we designed a cell competition assay where we could carefully quantify entosis, as well as proliferation and other forms of cell death, in mixed cultures treated with or without the ROCK inhibitor Y27632. The repetitive induction of entosis by cycles of suspension and adherence led to the progressive decrease in number of losers (MCF7, labeled with GFP), and increase in that of winners (VmCUB3, labeled with mCherry), as quantified by fluorescence microscopy (Figure 2A and 2B) and western blotting (Figure 2C). This competition between tumor cells could be inhibited by Y27632 treatment, which blocks entosis (Figure 2). Importantly, Y27632 did not increase proliferation of loser cells, but rather slightly decreased it (Supplementary information, Figure S3A), and also had no significant effect on other forms of cell death measured 
A

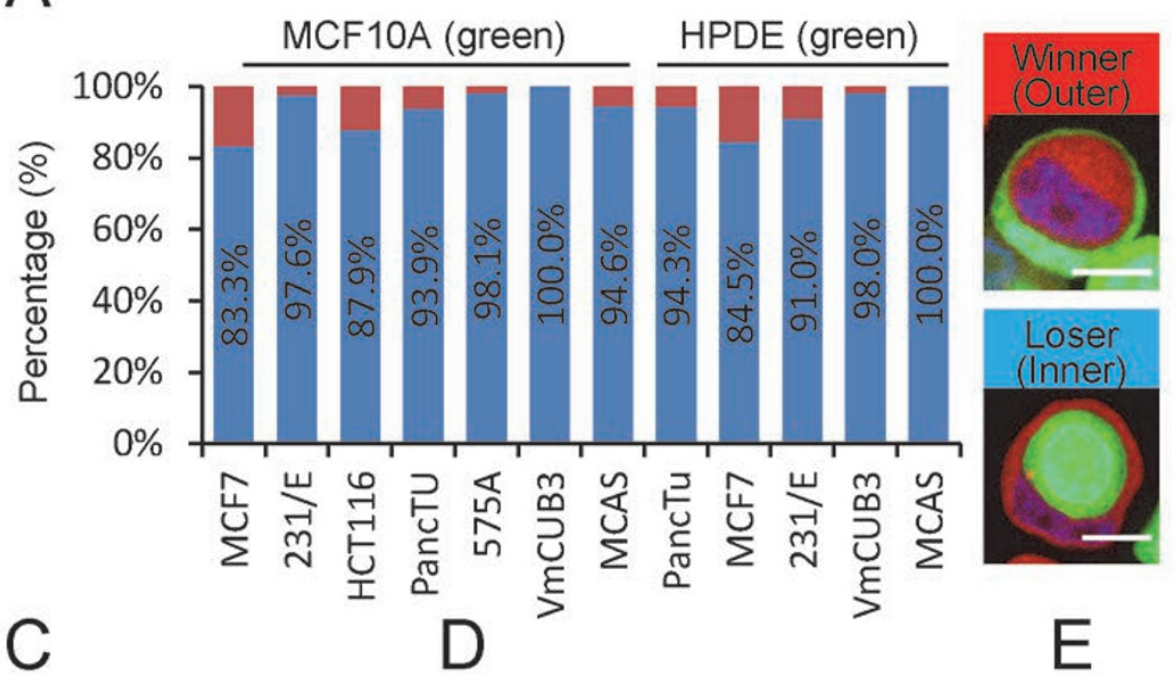

B

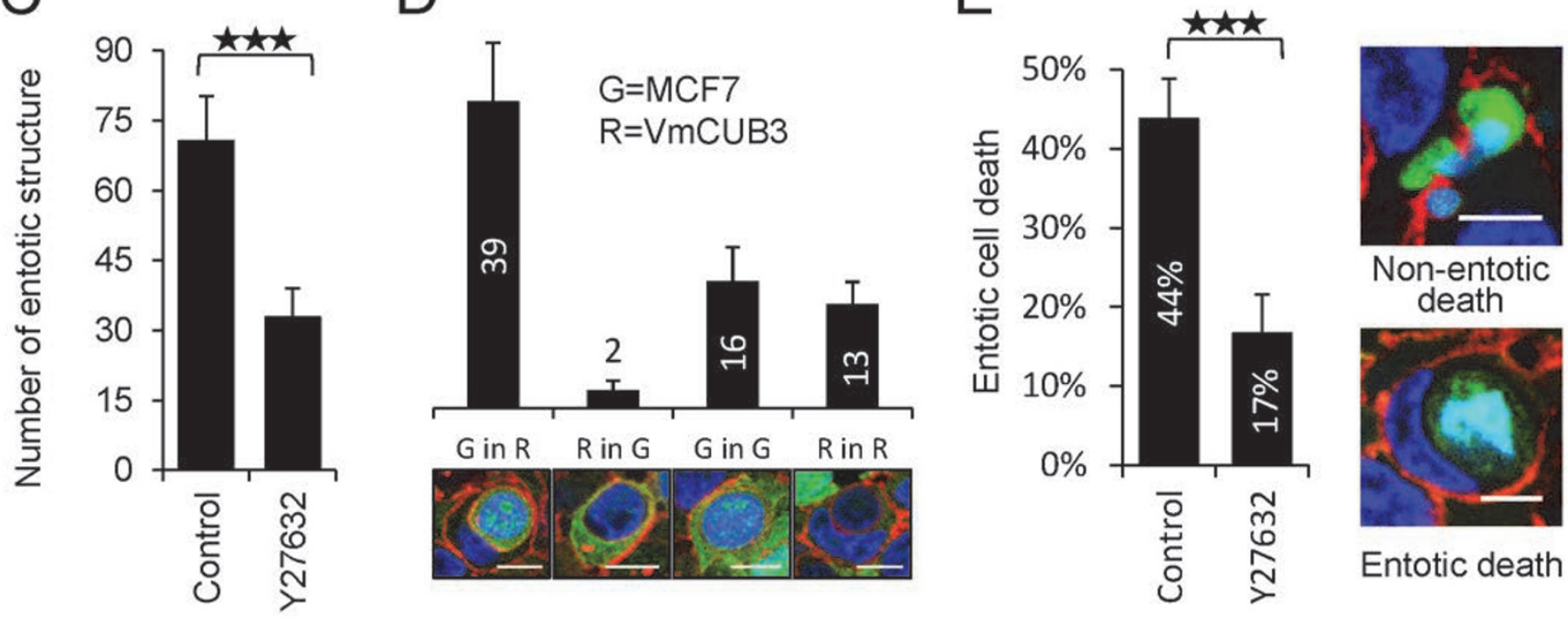

Figure 1 Entotsis-mediated cell competition in vitro and in vivo. (A) Position (winner or outer, and loser or inner) of non-transformed epithelial cells (MCF10A and HPDE cells labeled green) in entotic structures formed between MCF10A or HPDE cells and a range of tumor cells (red). MCF10A (mammary) and HPDE (pancreatic) are non-transformed human epithelial cells, and the remaining cell lines are human tumor cells: MCF7 is breast carcinoma cell line, MDA-MB-231/E is breast carcinoma cell line expressing exogenous E-cadherin, HCT116 is colon carcinoma cell line, PancTu is pancreatic carcinoma cell line, 575A and VmCUB3 are bladder carcinoma cell lines, and MCAS is ovarian carcinoma cell line. $n=42-77$ entotic structures. 231/E is MDA-MB-231 cells expressing E-cadherin. All other tumor cell lines express endogenous E-cadherin. Scale bar, $10 \mu \mathrm{m}$. (B) Representative image showing cell-in-cell structures (yellow arrowheads) formed between MCF7 and VmCUB3 cells. The cell-in-cell structure indicated by hatched box in the left image is enlarged and shown in individual fluorescent channels for MCF7 (CellTracker Green) and VmCUB3 (CellTracker Red) in right images. Scale bar, $20 \mu \mathrm{m}$. (C) Graph shows the average number of cell-in-cell structures from 20 high magnification fields ( $\times 63$ objective) of sections from mixed MCF7 and VmCUB3 xenograft tumors with or without Y27632 treatment. Data are mean \pm SD of different tumors; $P<0.001 . n=7$ for each group. (D) Graph shows calculation for cell engulfment events from C divided into four subcategories as indicated, where MCF7 cells expressed GFP (green = G) and VmCUB3 expressed mCherry (Red = R). Scale bar, $10 \mu \mathrm{m}$. Also see Supplementary information, Figure S2A, S2C and S2D. (E) Inhibition of entotic cell death in vivo by Y27632 treatment. Cell death, detected by TUNEL staining, was quantified from tumor sections with or without Y27632 treatment. TUNEL-positive nuclei enclosed within a cell were scored as entotic death, and TUNEL signals in between cells were scored as non-entotic death. Graph shows the percentage of entotic death out of all types of cell death. Data are mean \pm SD of different tumors; $P<0.001$. $n=6$ for each group. More than 45 TUNEL-positive cells were counted for each tumor sample. Also see Supplementary information, Figure S2B. 

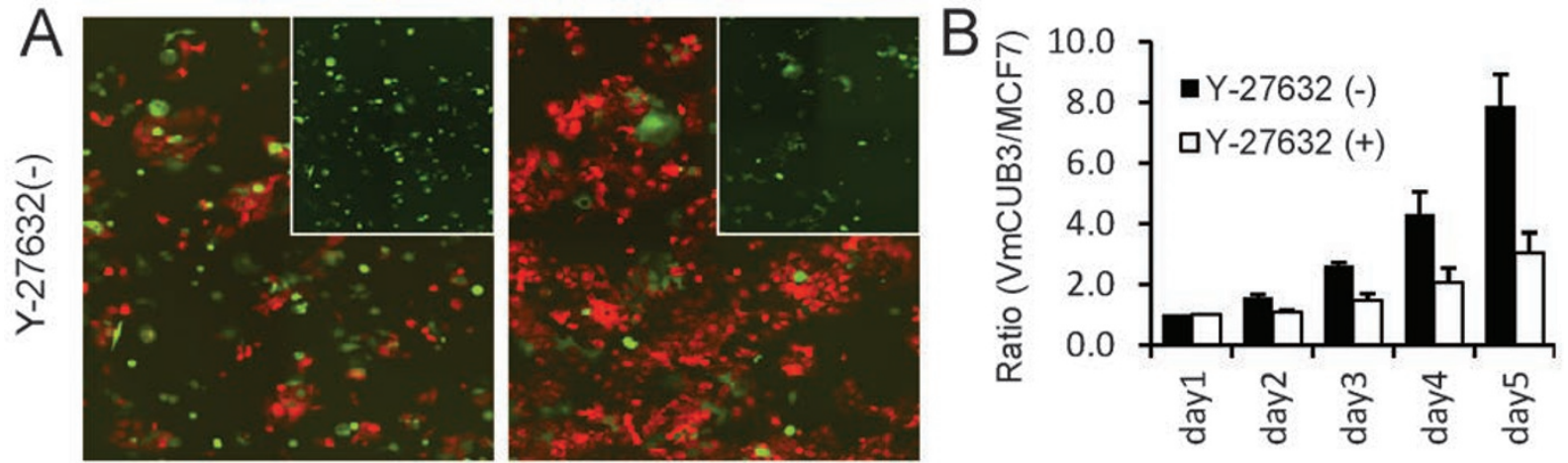

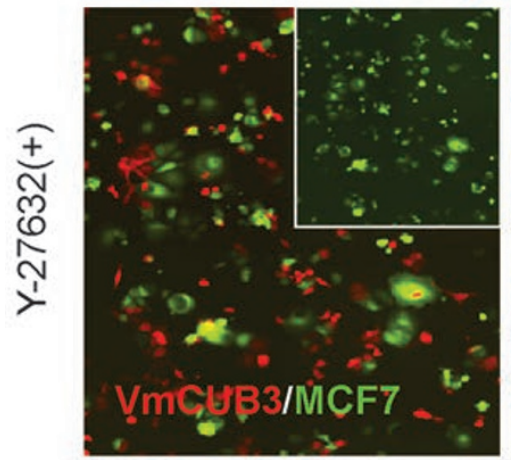

Day2

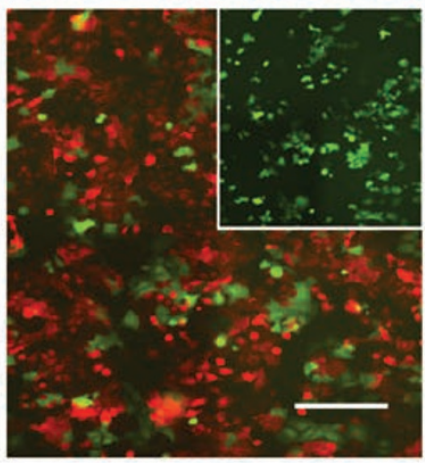

Day5
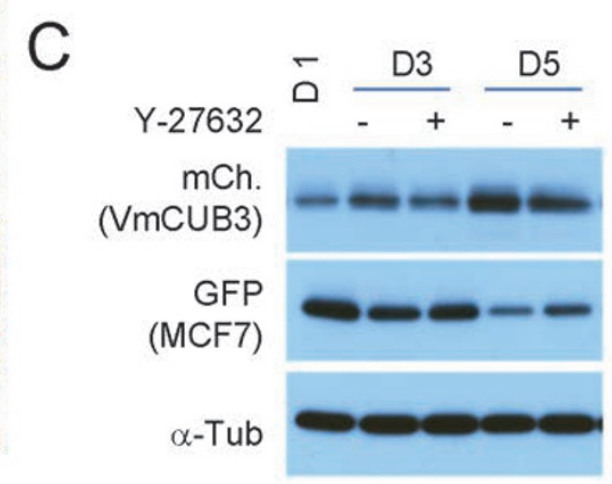

Figure 2 Winner cells outcompete losers by entosis in a mixed population. (A) Representative images of VmCUB3 (mCherry in red) and MCF7 (GFP in green) co-cultures with repetitive entosis induction in the presence or absence of $10 \mu \mathrm{M}$ Y27632. Insets show GFP fluorescence indicating MCF7 cells. Scale bar, $200 \mu \mathrm{m}$. (B) Fold changes of relative cell numbers between VmCUB3 and MCF7 upon repetitive entosis induction in the presence or absence of $10 \mu \mathrm{M}$ Y27632. Data are mean \pm SD of three experiments; $P<0.0072$ from day 2 to day 5. $n=7704-12341$ cells for each time point. (C) Accumulation of mCherry (VmCUB3) and loss of GFP (MCF7) with repetitive entosis induction. D1, 3, 5 represent days 1, 3, 5 from B.

by the incorporation of the nuclear dye DAPI into dead cells (Supplementary information, Figure S3B). These data suggest that entosis mediates a form of competition between human tumor cell populations.

\section{Winner cell status correlates with increased mechanical deformability}

We next attempted to investigate cell properties that may dictate winner versus loser status during entosis. We hypothesized that the accumulation of actomyosin at the cortex of loser cells, as shown in the companion paper by Sun et al. [18], would drive changes in cortical mechanics between daughter cells, which could dictate winner and loser cell status. Indeed, in MCF10A cultures loser (inner) cells were $45 \% \pm 8 \%$ less deformable than winner (outer) cells when interrogated by micropipette aspiration (MPA; Figure 3A and Table 1), demonstrating differential mechanical deformability between winners and losers, which is consistent with the loser-specific accumulation of actomyosin. Similarly, a recently published study also observed that loser cells exhibited greater rigidity than winner cells [19]. Overexpression of RhoA, ROCKI, or ROCKII was sufficient to confer loser status to cells mixed with normal cells, whereas inhibition of RhoA by overexpression of RhoA-N19 (dominant-negative mutant of RhoA) or the Rho-GTPase activating protein (GAP) p190A, showed the opposite effect (Figure 3B). These data suggest that the accumulation of actomyosin is ROCK-dependent (see a companion paper by Sun et al. [18]) and is sufficient to promote the uptake of losers. Notably, VmCUB3 tumor cells, which were found to be winners over MCF7, were almost two-fold more deformable than MCF7 cells (Figure 3C and Table 1 ), consistent with the model where mechanical deformability, a property controlled by Rho-ROCK signaling and actomyosin, dictates the identity of winner and loser cells during entosis.

Actomyosin contractility is a critical driving force of entosis

To further examine the observed differences in actomyosin between winner and loser cells, we used math- 
ematical modeling to investigate the role of mechanical force induced by contractile myosin at the cortex of loser cells in driving the cellular shape changes that are involved in entosis. As shown in Figure 4 (see also Supplementary information, Figure S4), energy landscapes for
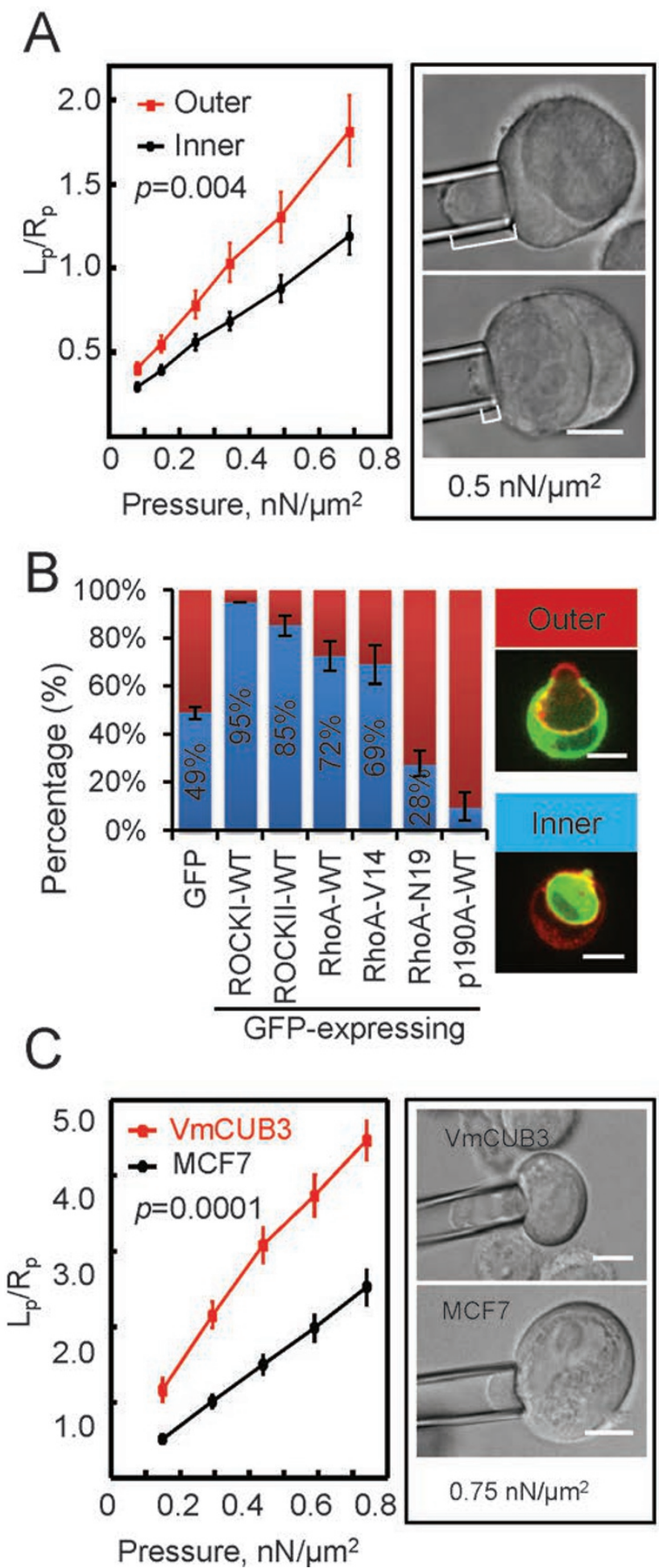

Pressure, $\mathrm{nN} / \mathrm{um}^{2}$ entosis with either early (Figure 4A) or late (Figure 4B) symmetry breaking (depending on the relative timing of the initiation of internalization between pairs of cells forming adhesions), indicate that myosin II functions to greatly reduces $\Delta E_{\text {total }}$ to negative values (i.e., $\Delta E_{\text {total }}^{<}$ 0 ), allowing entosis to proceed spontaneously. Actomyosin contractility within internalizing cells is therefore a critical driving force of entosis that is likely sufficient to promote cell shape changes associated with engulfment.

\section{Oncogenic Kras imparts winner status to cells}

To identify potential genetic determinants of winner status in tumor cells, we considered whether known pathways activated in tumor cells could modulate contractile myosin and influence entosis. Whereas non-tumorigenic cells were losers when paired with all tumor cell lines tested, we noted that MCF7 cells were losers when paired with cells with active mutant Kras (231/E and PancTu, Figure 5A). Expression of active mutant KrasV12 was sufficient to impart winner status to non-tumorigenic cells (Figure 5B), coincident with decreased pMLC2 levels (Figure 5C and Supplementary information, Figure S5A), suggesting that Kras activation may influence entosis by inhibiting actomyosin contraction. Indeed, expression of KrasV12 also increased the mechanical deformability of non-tumorigenic cells (Figure 5D and Table 1). Similarly, knockout of a mutant Kras allele in colon tumor cells increased pMLC2 levels (Figure 5E), as previously reported [20], and converted these mutant Kras-knockout cells into losers over their tumorigenic counterparts (Figure 5F). Together, these data demonstrate that the activation of Kras influences entosis by inducing winner status.

Figure 3 Relative Rho activity and cell deformability dictate winner and loser cell status. (A) Deformation of paired outer (upper image) and inner (lower image) cells measured by MPA with a pressure of $0.5 \mathrm{nN} / \mu \mathrm{m}^{2}$. Graph shows average deformation normalized by the radius of the micropipette measured by MPA with various levels of pressure. MCF10A cells were used in this experiment. Data are mean \pm SEM; $P=0.004(n=23)$. See Supplementary information, Data S1 for statistical analysis. Scale bar, $10 \mu \mathrm{m}$. (B) Relative position of MCF10A cells (green) expressing the indicated genes in entotic structures when mixed with control MCF10A cells. Data are mean \pm SD of three experiments, $n=99-319$ entotic structures. All differences from GFP control are statistically significant $(P<0.02)$. WT, wild type. Scale bar, $10 \mu \mathrm{m}$. (C) Deformation of MCF7 and VmCUB3 cells measured by MPA. Graph shows average deformation normalized by the radius of the micropipette. Data are mean $\pm \mathrm{SD} ; P=$ $0.0001 . n_{(\mathrm{VmCUB})}=16 ; n_{(\mathrm{MCF})}=17$. Image shows cells measured with a pressure of $0.75 \mathrm{nN} / \mu \mathrm{m}^{2}$. Scale bars, $10 \mu \mathrm{m}$. 
Table 1 Summary of measured mechanical parameters

\begin{tabular}{lll}
\hline Cell-in-cell & Elastic Modulus, $\mathrm{nN} / \mu \mathrm{m}^{2}$, Mean $\pm \mathrm{SEM}, n$ & $P$-value \\
\hline Figure 3A & & $0.098 \pm 0.013,23$ \\
Outer cell & $0.16 \pm 0.019,23$ & 0.004 \\
Inner cell & Elastic modulus, $\mathrm{nN} / \mu \mathrm{m}^{2}$, mean $\pm \mathrm{SEM}, n$ & $P$-value \\
\hline Cell line & & 0.0001 \\
\hline Figure 3C & $0.045 \pm 0.0036,16$ \\
VmCUB3 & $0.089 \pm 0.013,17$ \\
MCF7 & \\
\hline Figure 5D & $0.052 \pm 0.0082,9$ \\
Kras & $0.10 \pm 0.015,10$ \\
MCF10A &
\end{tabular}

${ }^{1}$ Mann-Whitney-Wilcoxon rank sum test

${ }^{2}$ ANOVA with Fisher's least significant difference post hoc test

Racl is required for KrasV12-induced winner status

As Ras signaling is known to induce Rac1 activation, we examined the effect of Rac1 activation on entosis. Rac1 was required for winner status induced by KrasV12 in non-transformed cells, as shRNA-mediated knockdown of Rac1 partially reverted KrasV12-induced phenotype (Figure 6A). Like KrasV12, constitutive-active Rac1(V12) decreased pMLC2 levels (Figure 6E and Supplementary information, Figure S5B) and conferred winner status to cells (Figure 6B). Conversely, dominant-negative Rac1N17-expressing cells were shifted toward loser status (Figure 6B). Similarly, the majority of Rac1-knockdown cells, which exhibited increased levels of pMLC2, became losers in a ROCKI/II-dependent manner (Figure 6C, 6D and Supplementary information, Figure S5C-S5D).

Altogether, these data fit a model where the setpoint of contractile myosin dictates engulfed cell identity during entosis. Activated Ras/Rac signaling downregulates ROCK/Rho activity and actomyosin contractility, promoting competitive cell engulfment (Supplementary information, Figure S6).

\section{Discussion}

In this study, we reveal a mode of cell competition occurring between human cells, which involves cell engulfment and killing. Competition by entosis, a mechanism of cell cannibalism, mediates the killing of "loser" cells by "winners" in mixed cell populations. We find that differences in mechanical deformability between epithelial cadherin-expressing cells determine which cells "win" in this program of competitive engulfment. It has been reported previously that tumor progression is associated with decreased cortical tension or increased mechanical deformability of tumor cells [21-23], a property that may facilitate metastasis by promoting cell invasion away from primary tumors [24]. We find that expression of constitutively active Kras mutant leads to activation of Rac1, which subsequently increases mechanical deformability in epithelial cells, similar to previous observations in fibroblasts [25]. While Rac1 is required for Kras to induce winner status, we cannot rule out the possibility that other pathways engaged by Kras activation, such as MAP kinase signaling, PI-3-kinase, or even autophagy, could also participate in regulating entosis. Our data also demonstrate that highly deformable tumor cells ingest neighboring tumor cells that are less deformable, which could contribute to selection for oncogenic insults that increase cell deformability within heterogeneous tumor cell populations. Human tumors are known to be mechanically heterogeneous [26], which may also prime tumor cell populations for entosis.

As discussed in the companion paper by Sun et al. [18], cytoskeletal tension associated with entosis involves RhoA activity and contractile myosin that are positioned in patterns resembling those previously reported between pairs of adherent cells forming cell-cell junctions [27], suggesting that entosis results from imbalances of otherwise normal cell-cell adhesion-associated forces. The force imbalance alone could be sufficient to further activate myosin II contractility and promote entosis $[28$, 29]. The activation of RhoA in one cell of a pair, or introduction of phosphomimetic MLC, has been shown to induce growth of adherens junction as a result of increased tugging force by actomyosin [30]. Here, we show a similar effect of tension imbalance that promotes junctional growth that is sufficient to enwrap an entire cell. It is conceivable that such imbalances in cell deformability may occur normally between adherent cells, but the 
A
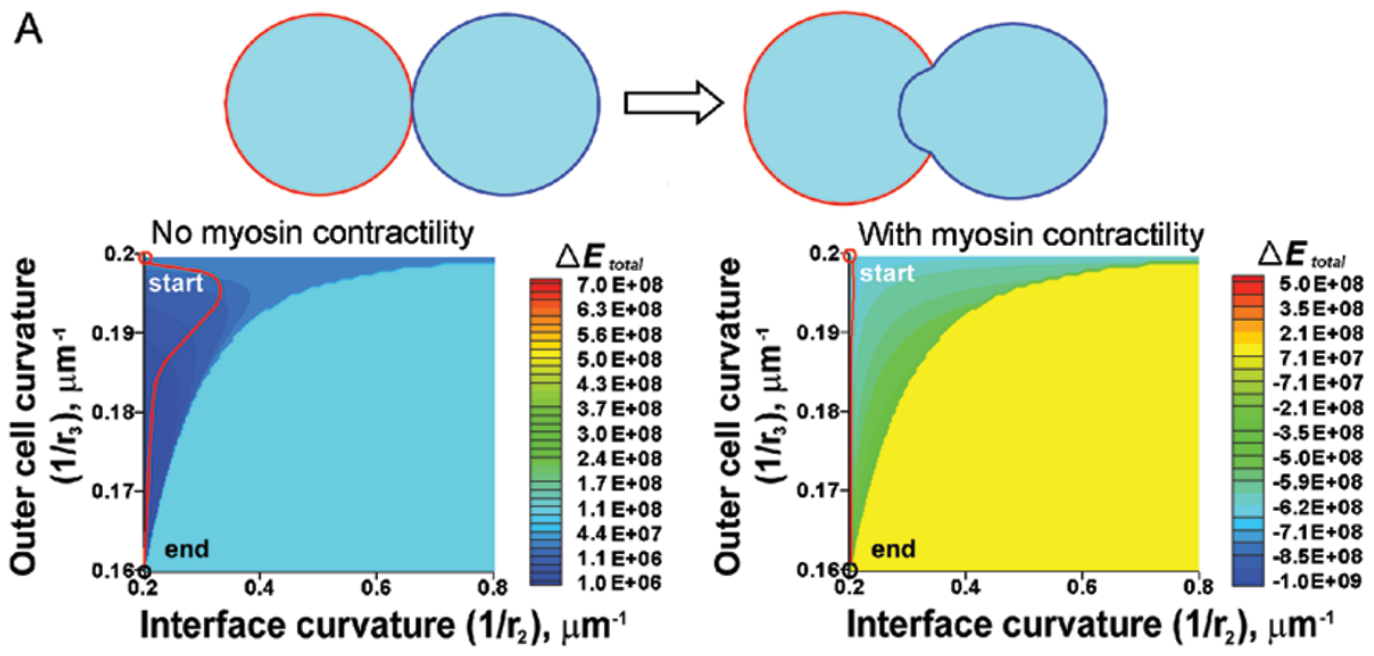

B
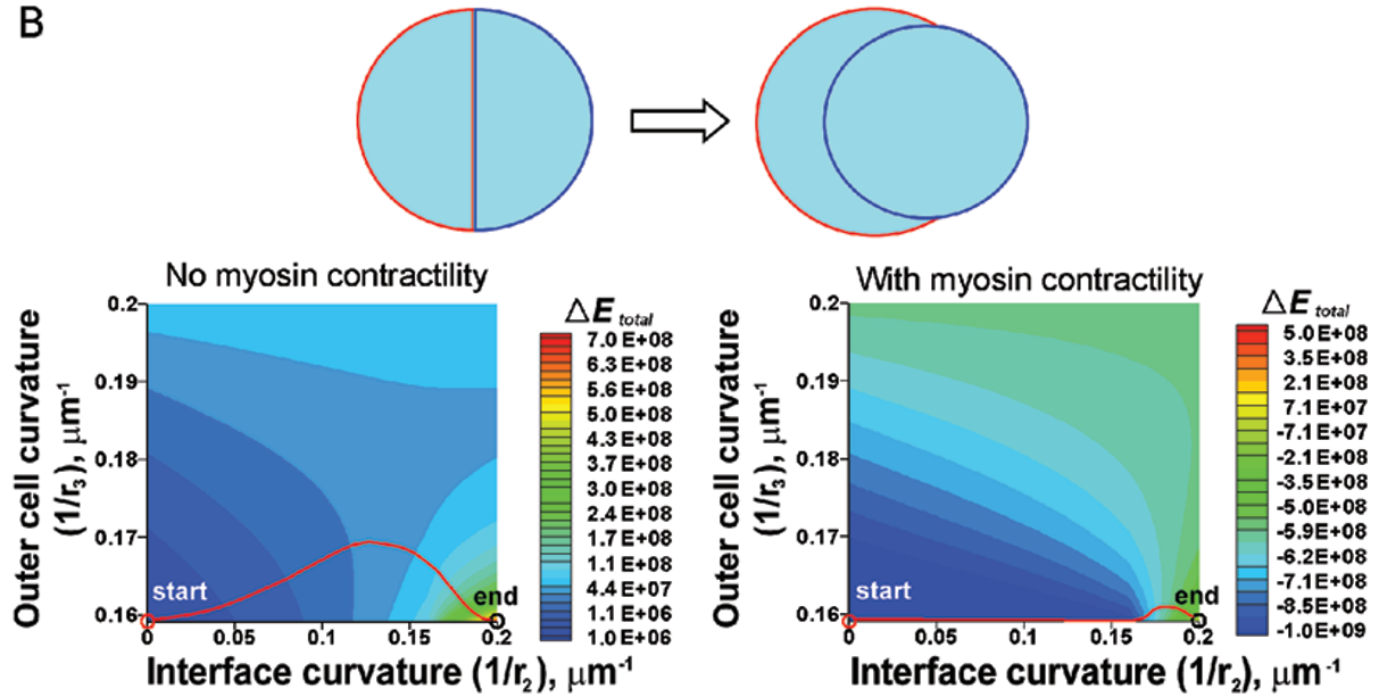

Figure 4 Energy landscapes for entosis. (A) Energy landscapes for entosis during early symmetry breaking. (B) Energy landscapes for entosis during late symmetry breaking. The left contour plots have no myosin contractility while the right contour plots include a myosin II contractility difference between inner and outer cells. Red and black circles represent the start and completion of entosis, respectively. The red connecting lines represent the minimum energy paths. These lines describe how the geometrical parameters $r_{2}$ and $r_{3}$ change over the course of entosis. Initially, in $\mathbf{A} \overline{r_{1}}=\overline{r_{2}}=\overline{r_{3}}=5 \mu \mathrm{m}$ while in B $\bar{r}_{1}=\bar{r}_{3}=5 \sqrt[3]{2} \mu \mathrm{m}$ and $\bar{r}_{2}=\infty$. The remaining parameters are the same for both panels: $k_{1}=k_{2}=400 \mathrm{k}_{\mathrm{B}} \mathrm{T}, D_{1}=2 \times 10^{4} \mathrm{k}_{\mathrm{B}} \mathrm{T}$ (derived from Figure 3A), $D_{1}=1 \times 10^{4} \mathrm{k}_{\mathrm{B}} \mathrm{T}$ (derived from Figure $3 \mathrm{~A}$ ), $\rho=100$ molecules $/ \mu \mathrm{m}^{2}, \varepsilon=20 \mathrm{k} \mathrm{T}, \gamma=0, N=1000 \mathrm{~mol}-$ ecules $/ \mu \mathrm{m}^{2}, \chi=0.8$, and $f=4 \mathrm{pN}$. In $\mathbf{A}$, the areas with uniform color are inaccessible because the cells begin to lose contact. Similarly, in $\mathbf{B}$, regions where both cells have high curvature are also inaccessible.

counterbalancing effect of cell-matrix adhesion blocks cell engulfment, as proposed previously [13].

Our previous reports $[13,17]$ and data presented in the companion paper by Sun et al. [18] demonstrate that entosis may suppress transformed growth by inducing cell death; however, entosis could also promote tumor progression by inducing ploidy changes [16] and supplying engulfing cells with nutrients [31]. Human tumors are known to be genetically heterogeneous, as many are composed of multiple independent clones with genetic alterations such as changes in ploidy [32]. Entosis could allow neighboring cells to compete by acting as a mechanism of cell death of losers, while endowing winners with ploidy changes that promote tumorigenesis. Indeed, we and others have shown that "cell-in-cell" structures in human tumors, which resemble those formed by entosis, occur most frequently in high-grade, aggressive breast tumors that are known to exhibit high rates of aneuploidy 

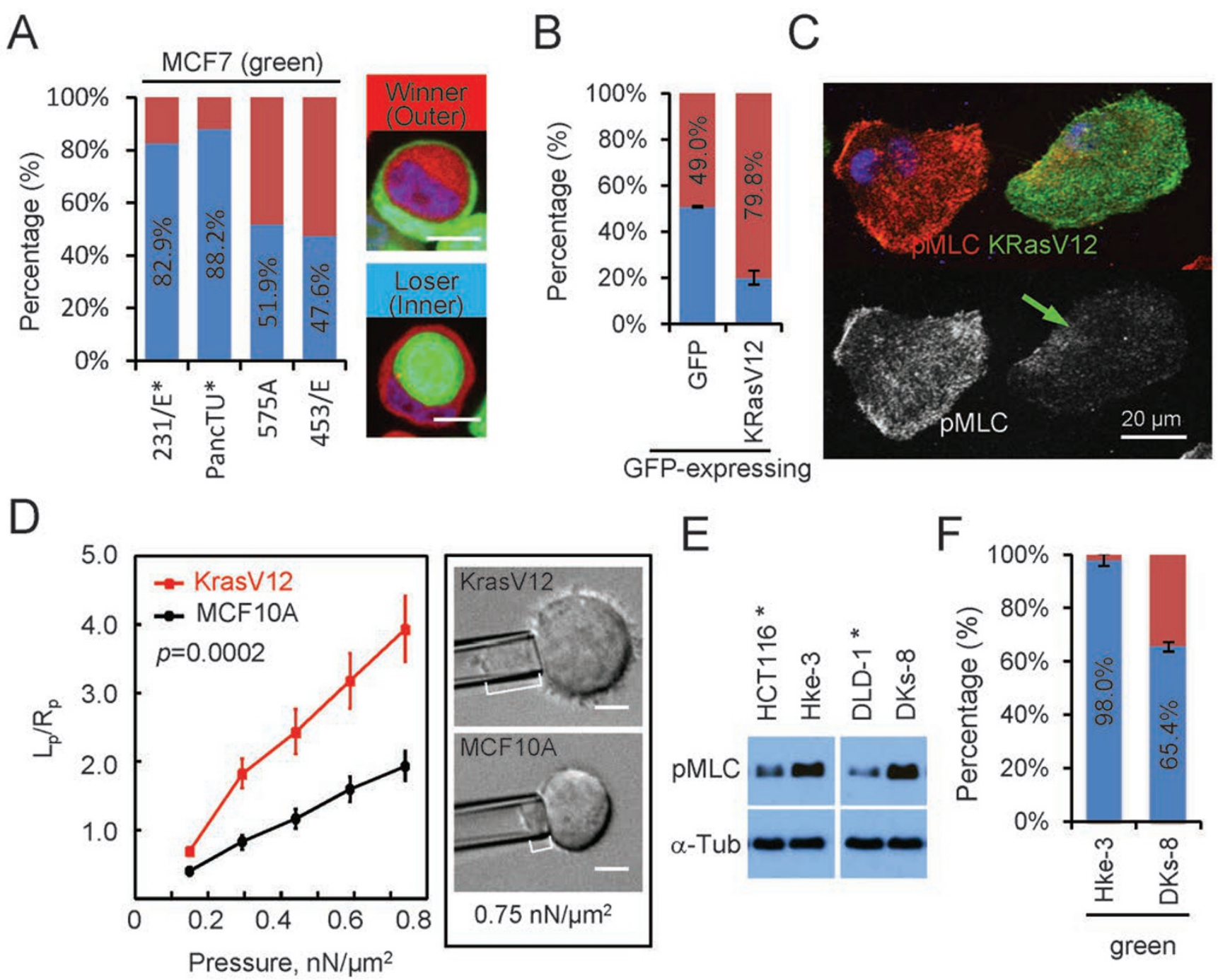

Figure 5 Oncogenic Kras imparts winner status to cells. (A) Position of MCF7 cells (green) in entotic structures formed between MCF7 cells and other tumor cells (red). $n=70-108$. Kras-mutant cell lines are indicated with *. Scale bars, $10 \mu \mathrm{m}$. (B) Expression of KrasV12 (green) imparts winner status to non-transformed MCF10A cells over controls. Data are mean \pm SD of three experiments, $n=146-151$ entotic structures. (C) Inhibition of pMLC2 by GFP-KrasV12 expression. Arrow indicates GFP-KrasV12-expressing cell. Scale bars, $20 \mu \mathrm{m}$. Also see Supplementary information, Figure S5A for immunoblotting. (D) Deformation of KrasV12-expressing MCF10A versus control cells measured by MPA. Graph shows average deformation normalized by the radius of the micropipette. Data are mean $\pm \mathrm{SD} ; P=0.0002 . n_{(\mathrm{MCF} 10 \mathrm{~A})}=10 ; n_{(\mathrm{KrasV} 12)}=9$. Image shows cells measured with a pressure of $0.75 \mathrm{nN} / \mu \mathrm{m}^{2}$. Scale bars, $10 \mu \mathrm{m}$. (E) pMLC2 expression levels compared between isogenic cell pairs with mutant (HCT116 and DLD-1) or wild-type (Hke-3 and Dks-8) Kras. (F) Position of Hke-3 and Dks-8 cells (wild-type Kras, green) in entotic structures formed between Hke-3 and Dks-8 cells and their isogenic cells (mutant Kras), HCT116 and DLD-1, respectively. Data are mean \pm SD of triplicate experiments. $n_{(\mathrm{Hke}-3)}=67, n_{(\mathrm{Dks}-8)}=139$ entotic structures.

$[16,33]$. While the mechanisms underlying the formation of such "cell-in-cell" structures in human tumors remain controversial [14], we show here that the engulfment and killing of loser cells by winners, which is dependent on epithelial cadherin expression and ROCK activity in culture, also occurs between tumor cells in xenografts. Further, this cell cannibalism is reduced by treating mice with the ROCK inhibitor Y27632, providing evidence that entosis indeed promotes cell-in-cell formation in vivo.

Like cell competition defined in Drosophila, engulfment by entosis allows cells to outcompete neighbors of different genotypes. However, this entotic mechanism has several notable distinctions. First, cell death by entosis is generally non-apoptotic, whereas losing clones in Drosophila die by apoptosis [34]. Second, we observe 

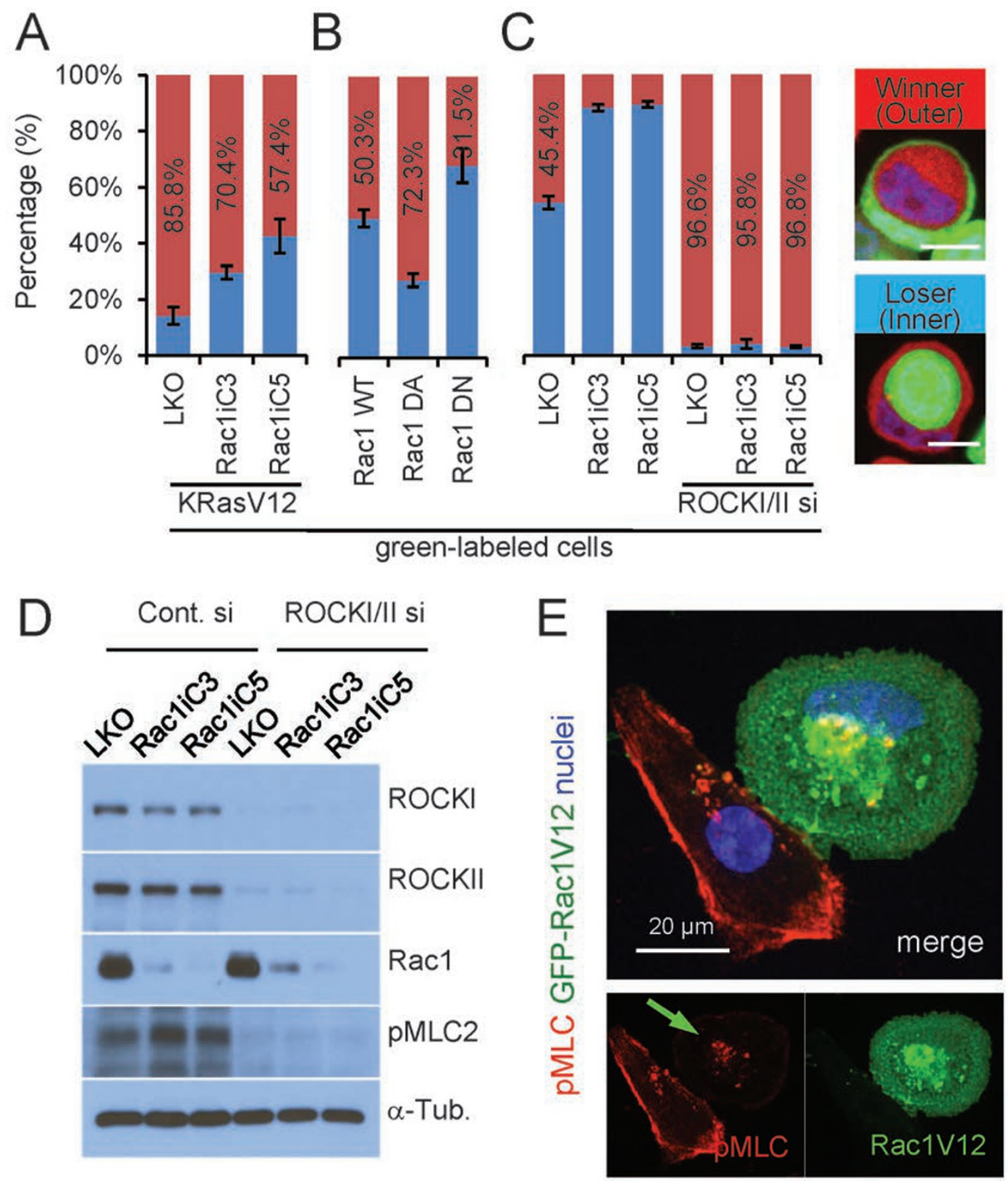

Figure 6 Rac1 is required for oncogenic Kras-induced winner identity. (A) Rac1 knockdown (Rac1iC3 and Rac1iC5 with LKO as control) inhibits winner status induced by GFP-KrasV12 in non-transformed MCF10A cells. $P<0.05$ for Rac1iC3 compared to LKO; $P<0.001$ for Rac1iC5 compared to LKO. (B) Activation or inhibition of Rac1 changes the identity of green-labeled cells during entosis. WT, wild type; DA, dominant active; DN, dominant negative. (C) Reversal of Rac1-knockdown cells (green) from loser into winner status by ROCKI/II co-depletion. For A-C, data are mean \pm SD of more than 3 experiments. $n$ = 99-238 entotic structures. Scale bar, $10 \mu \mathrm{m}$. (D) Immunoblotting of pMLC2 after Rac1 and ROCKI/II depletion. (E) Inhibition of pMLC2 by GFP-Rac1V12 expression. Scale bar, $20 \mu \mathrm{m}$. Arrow indicates GFP-Rac1V12-expressing cell. Also see Supplementary information, Figure S5B for immunoblotting.

that neighboring cell engulfment mediates competition between human cells. This observation is in contrast to a recent report which suggests that in Drosophila tissues, the post-apoptotic engulfment of loser cells may be mediated by hemocytes, which phagocytize cell corpses, rather than by the neighboring cells [35]. While the identity of the engulfing cells and whether engulfment itself mediates competition in Drosophila are controversial, we clearly show that neighboring cell-mediated engulfment and killing occur in mixed populations of human cells. 
Other mechanisms of competition between mammalian cells have been demonstrated, including the differential extrusion of cells of mixed genotypes from monolayer cultures $[9,10]$, competition between cells of the early embryo [36-38], and competition between hematopoietic progenitors driven by differences in p53 activity $[39,40]$. Perhaps diverse competition mechanisms reflect a stringent requirement for homeostasis in different contexts within various tissue types of metazoan organisms during development and adulthood. To our knowledge, our data are the first to demonstrate a mechanism of direct competition between mammalian cells, which utilizes cell engulfment that is dependent on cell adhesion and differential cell mechanics to allow winners to kill losers.

\section{Materials and Methods}

\section{Cells and culture conditions}

MCF7, MCAS, PancTu, VmCUB3, 575A, HCT116, HKe-3 [41], DLD1, DKs-8, and 293FT cells were maintained in Dulbecco's modified Eagle's medium (DMEM) supplemented with $10 \%$ fetal bovine serum (FBS, Sigma-Aldrich). MDA-MB-231, MDAMB-453 and their derivative cell lines were maintained in Leibovitz's L-15 (Invitrogen) supplemented with 10\% FBS. MCF10A cells were cultured in DMEM/F12 supplemented with 5\% horse serum (Atlanta Biologicals), $20 \mathrm{ng} / \mathrm{ml}$ EGF (Peprotech), $10 \mu \mathrm{g} / \mathrm{ml}$ insulin (Sigma), $0.5 \mu \mathrm{g} / \mathrm{ml}$ hydrocortisone (Sigma), and $100 \mathrm{ng} /$ $\mathrm{ml}$ cholera toxin (Sigma). HPDE-E6E7 cells were cultured in keratinocyte serum-free medium supplemented with bovine pituitary extract and EGF (Invitrogen).

\section{Antibodies and chemical reagents}

Antibodies with working dilution factors, company source and catalog number include: anti-mCherry (1:500; Clontech; 632496), anti-GFP (1:500 or 1:200; Invitrogen; A11122 and G-10362), anti-tubulin (1:5 000; Sigma; T5168), anti-pMLC (1:200 or 1 000; Cell Signaling; \#3671), anti-E-cadherin (1:200 or 1:1 000; BD Biosciences; BDB610181), anti- $\beta$-catenin (1:200 or 1:1 000; Sigma; C2206), anti-Rac1 (1:500; Upstate; 05-389), anti-ROCK I (1:200; Santa Cruz; sc-6056), anti-ROCK II (1:200; Upstate; 07443), and anti-BrdU (1:200; Cell Signalling; \#5292). Secondary antibodies include Alexa Fluor 568 anti-rabbit (1:300; Invitrogen; A11036) and Alexa Fluor 488 anti-mouse (1:300; Invitrogen; A11029). The ROCK inhibitor Y27632 was purchased from TOCRIS (1254) and used at final concentration of $10 \mu \mathrm{M}$. DAPI was purchased from Sigma (D8417). BrdU was purchased from Sigma (B5002) and used at final concentration of $10 \mu \mathrm{M}$.

\section{Constructs}

pEGFP-Rac1-WT, -DA, -DN, pEGFP-RhoA-WT, -V14, and -N19 [42] were gifts from Dr Alan Hall (MSKCC, USA). pQCXIP-GFP and pQCXIP-GFP-KRasV12 were gifts from Ms Kirti Magudia from the lab of Dr Alan Hall. pQCXIP-mCherry was a gift from Ms Yuji Shi from the lab of Dr Xuejun Jiang (MSKCC, USA). pEGFP-p190A WT [43] was a gift from Dr Keith Burridge (UNC, USA). The RNA interference hairpin constructs for human Rac1 (Rac1iC3 and Rac1iC5) were gifts from Dr Joan Brugge.
Virus production and infection

Viruses were produced as previously described [13]. Briefly, 1 $\times 10^{6}$ 293FT cells were plated into 6-well plates coated with poly-D-lysine. Transfection was performed with lenti- or retro-viral constructs together with packaging plasmids, and viruses were collected twice at 24-h intervals. To infect cells, $1 \mathrm{ml}$ of viral supernatants mixed with $1 \mu \mathrm{l}$ of polybrene from a $10 \mu \mathrm{g} / \mathrm{ml}$ stock was added to target cells for $12 \mathrm{~h}$, followed by feeding with regular media. Cells were selected with puromycin $(2 \mu \mathrm{g} / \mathrm{ml})$ or G418 (200 $\mu \mathrm{g} / \mathrm{ml})$. Fluorescent protein-expressing cells were sorted by flow cytometry if needed.

\section{Transfection and nucleofection}

siGenome SMART pool siRNAs against human ROCKI and ROCKII were obtained from Dharmacon. For siRNA transfection, $1 \times 10^{5}$ MCF10A cells were transfected with $100 \mathrm{nM}$ siRNA using Oligofectamine (Invitrogen). Cell analyses were performed $48 \mathrm{~h}$ post transfection. For nucleofection, $1 \times 10^{6} \mathrm{MCF} 10 \mathrm{~A}$ cells were transfected with $6 \mu \mathrm{g}$ of target plasmids by program T-24 using Nucleofector Solution V reagent (Lonza), and cell analyses were performed $12 \mathrm{~h}$ post nucleofection.

\section{Quantification of cell-in-cell structures and winner/loser cell identity}

To quantify cell-in-cell structures, cells were cultured in suspension for $6 \mathrm{~h}$ on Ultra-low Attachment plates (Corning) and then mounted onto glass slides by cytospin preparation and fixation in $10 \%$ trichloroacetic acid (TCA). Samples were immunostained with E-cadherin and $\beta$-catenin antibodies and analyzed by confocal microscopy as described [44]. Cell structures with more than half of cell body internalized were counted. For identity analysis of winners and losers, equal numbers of cells from two cell lines, stained with green or red CellTrackers (Invitrogen), were mixed together and cultured in suspension, and then plated into $0.4 \%$ soft agar or cytospun onto glass slides for analysis by confocal microscopy. To assess the effects of gene overexpression on cell identity, MCF-10A-mCherry-CAAX cells were nucleofected with constructs expressing GFP-tagged genes and cultured overnight, then placed into suspension for 3-4 h, and plated into $0.4 \%$ agar-containing medium for analysis by confocal microscopy. Structures formed between GFP-expressing and -non-expressing cells were analyzed.

\section{Immunostaining and immunoblotting}

For immunostaining [45], cells were fixed with 4\% PFA or $10 \%$ TCA, permeabilized with $0.2 \%$ Triton X-100/PBS for 5 min followed by blocking with 5\% BSA for $1 \mathrm{~h}$, and were incubated with primary antibodies in a humidified box overnight, followed by three 10-min washes with PBS. Secondary antibodies were applied for $1 \mathrm{~h}$ followed by three 10-min washes with PBS, and cells were counter-stained with DAPI and mounted with Prolong Gold antifade reagent (Invitrogen). For BrdU staining, additional procedures of denaturing with $2 \mathrm{~N} \mathrm{HCl}$ for 20 min and quenching with $0.1 \mathrm{M} \mathrm{Na}_{2} \mathrm{~B}_{4} \mathrm{O}_{7}$ for 2 min were performed before blocking. Immunoblotting was performed as previously described [46].

\section{Cell competition assay}

MCF7 cells expressing GFP and VmCUB3 cells expressing mCherry were mixed in suspension for $7 \mathrm{~h}$ with or without 
Y27632, and then plated onto adherent cultures overnight in the absence of Y27632, followed by quantification of relative cell numbers by microscopy, or preparation of cell lysates for western blotting, the next morning. This procedure was repeated for the indicated number of cycles.

\section{Xenograft tumor assay}

Female 6-8-week-old SCID mice were ovariectomized and embedded with $\beta-17$ estradiol pellets 3 days before tumor implantation. Cells with $70 \%-80 \%$ confluence were trypsinized and counted, and equal numbers of MCF7 cells expressing GFP and VmCUB3 cells expressing mCherry were mixed before injection. Ten million total cells per tumor (mixture of MCF7 and VmCUB3 at 1:1 ratio) were injected subcutaneously, and Y27632 (10 $\mu \mathrm{g} /$ $\mathrm{kg}$ ) was administered on day 1 after cells were implanted and once daily thereafter. Tumors were collected on day 5 for sectioning. Tumor samples were fixed in 4\% PFA for $2 \mathrm{~h}$ and then processed for paraffin embedding. Sections were deparaffinized as previously described [47] and retrieved in Tris-EDTA-Tween-20 (0.05\%) solution in $100{ }^{\circ} \mathrm{C}$ water bath for $20 \mathrm{~min}$ before proceeding to TUNEL and immunostaining. Cell engulfments were identified by confocal microscopic examination of $10 \mu \mathrm{m}$ tumor sections immunostained for GFP and E-cadherin. To verify that the cell engulfments identified in $10 \mathrm{~m}$ sections represent engulfment activity in 3-dimensions, engulfments were also examined in thick $(30 \mu \mathrm{m})$ tumor sections through confocal z-planes. $91 \%$ of engulfments identified at the midplane of $30 \mu \mathrm{m}$ sections contained at least $2 / 3$ of one-cell-body internalized into another in 3-dimensions (Supplementary information, Figure S1D and S1E).

\section{TUNEL assay}

The ApopTag Plus Peroxidase In Situ Apoptosis Detection Kit (Millipore, S7101) was used to detect cell death in tumor sections according to the manufacturer's instructions. Briefly, antigen-retrieved sections were equilibrated and applied with TdT enzyme in a humidified chamber at $37^{\circ} \mathrm{C}$ for $1 \mathrm{~h}$. Reactions were stopped by incubating specimens in stop/wash buffer for $10 \mathrm{~min}$ before proceeding to standard immunostaining procedure as described above.

\section{$M P A$}

MPA was performed as previously described [48], on intermediate entotic structures of MCF10A cells or single cells as indicated in suspension. For data analysis, the length of the deformation of cortex pulled into the micropipette $\left(L_{\mathrm{p}}\right)$ was normalized by the pipette radius $\left(R_{\mathrm{p}}\right)$, and $L_{\mathrm{p}} / R_{\mathrm{p}}$ values were plotted as a function of applied pressure. These data were converted into apparent elastic moduli [49] (Table 1).

\section{Statistics}

$P$ values were calculated by a two-tailed Student's $t$-test using Excel or GraphPad Prism 5 software. ANOVA with Fisher's least significant difference post hoc test and Wilcoxon sign-rank test were performed using Kaleidagraph software.

\section{Acknowledgments}

We thank Drs Alan Hall, Xuejun Jiang and their lab members and members of the Overholtzer laboratory for reagents and discussions on the manuscript. We thank Drs Keith Burridge, Klaus
Hahn, Marilyn Resh and Tingchao Chou for reagents. We thank Drs Elisa de Stanchina, Xiaodong Huang and Juan Qiu for help with animal experiments, and Ms Ning Fan for histology. This work was supported by the NCI (CA154649, MO), the NIGMS (GM66817, DNR), the Louis V Gerstner, Jr Young Investigators Fund (MO), the Benjamin Friedman Research Fund (MO), Cancer Research UK fellowship (C47718/A16337, OF), the National Basic Research Program of China (2015CB553704, QS), and the National Natural Science Foundation of China (30871364 and 81472588, QS).

\section{References}

1 Simpson P, Morata G. Differential mitotic rates and patterns of growth in compartments in the Drosophila wing. Dev Biol 1981; 85:299-308.

2 Morata G, Ripoll P. Minutes: mutants of Drosophila autonomously affecting cell division rate. Dev Biol 1975; 42:211221.

3 Moreno E, Basler K. dMyc transforms cells into super-competitors. Cell 2004; 117:117-129.

4 de la Cova C, Abril M, Bellosta P, Gallant P, Johnston LA. Drosophila myc regulates organ size by inducing cell competition. Cell 2004; 117:107-116.

$5 \mathrm{Li} \mathrm{W}$, Baker NE. Engulfment is required for cell competition. Cell 2007; 129:1215-1225.

6 Ohsawa S, Sugimura K, Takino K, et al. Elimination of oncogenic neighbors by JNK-mediated engulfment in Drosophila. Dev Cell 2011; 20:315-328.

7 Moreno E. Is cell competition relevant to cancer? Nat Rev Cancer 2008; 8:141-147.

8 Baker NE, Li W. Cell competition and its possible relation to cancer. Cancer Res 2008; 68:5505-5507.

9 Kajita M, Hogan C, Harris AR, et al. Interaction with surrounding normal epithelial cells influences signalling pathways and behaviour of Src-transformed cells. J Cell Sci 2010; 123:171-180.

10 Hogan C, Dupre-Crochet S, Norman M, et al. Characterization of the interface between normal and transformed epithelial cells. Nat Cell Biol 2009; 11:460-467.

11 Norman M, Wisniewska KA, Lawrenson K, et al. Loss of Scribble causes cell competition in mammalian cells. $J$ Cell Sci 2012; 125(Pt 1):59-66.

12 Tamori Y, Bialucha CU, Tian AG, et al. Involvement of Lgl and Mahjong/VprBP in cell competition. PLoS Biol 2010; 8:e1000422.

13 Overholtzer M, Mailleux AA, Mouneimne G, et al. A nonapoptotic cell death process, entosis, that occurs by cellin-cell invasion. Cell 2007; 131:966-979.

14 Sharma N, Dey P. Cell cannibalism and cancer. Diagn Cytopathol 2011; 39:229-233.

15 Overholtzer M, Brugge JS. The cell biology of cell-in-cell structures. Nat Rev Mol Cell Biol 2008; 9:796-809.

16 Krajcovic M, Johnson NB, Sun Q, et al. A non-genetic route to aneuploidy in human cancers. Nat Cell Biol 2011; 13:324330 .

17 Florey O, Kim SE, Sandoval CP, Haynes CM, Overholtzer M. Autophagy machinery mediates macroendocytic processing and entotic cell death by targeting single membranes. Nat 
Cell Biol 2011; 13:1335-1343.

18 Sun Q, Cibas ES, Huang H, Hodgson L, Overholtzer M. Induction of entosis by epithelial cadherin expression. Cell Res 2014; 24:1288-1298.

19 Xia P, Zhou J, Song X, et al. Aurora A orchestrates entosis by regulating a dynamic MCAK-TIP150 interaction. $J$ Mol Cell Biol 2014; 6:240-254.

20 Pollock CB, Shirasawa S, Sasazuki T, Kolch W, Dhillon AS. Oncogenic $K-R A S$ is required to maintain changes in cytoskeletal organization, adhesion, and motility in colon cancer cells. Cancer Res 2005; 65:1244-1250.

21 Ward KA, Li WI, Zimmer S, Davis T. Viscoelastic properties of transformed cells: role in tumor cell progression and metastasis formation. Biorheology 1991; 28:301-313.

22 Cross SE, Jin YS, Rao J, Gimzewski JK. Nanomechanical analysis of cells from cancer patients. Nat Nanotechnol 2007; 2:780-783.

23 Guck J, Schinkinger S, Lincoln B, et al. Optical deformability as an inherent cell marker for testing malignant transformation and metastatic competence. Biophys $J$ 2005; 88:36893698.

$24 \mathrm{Xu} \mathrm{W}$, Mezencev R, Kim B, et al. Cell stiffness is a biomarker of the metastatic potential of ovarian cancer cells. PLoS One 2012; 7:e46609.

25 Anderson KW, Li WI, Cezeaux J, Zimmer S. In vitro studies of deformation and adhesion properties of transformed cells. Cell Biophys 1991; 18:81-97.

26 Plodinec M, Loparic M, Monnier CA, et al. The nanomechanical signature of breast cancer. Nat Nanotechnol 2012; 7:757765.

27 Yamada S, Nelson WJ. Localized zones of Rho and Rac activities drive initiation and expansion of epithelial cell-cell adhesion. J Cell Biol 2007; 178:517-527.

28 Ren Y, Effler JC, Norstrom M, et al. Mechanosensing through cooperative interactions between myosin II and the actin crosslinker cortexillin I. Curr Biol 2009; 19:1421-1428.

29 Luo T, Mohan K, Srivastava V, et al. Understanding the cooperative interaction between myosin II and actin cross-linkers mediated by actin filaments during mechanosensation. Biophys $J$ 2012; 102:238-247.

30 Liu Z, Tan JL, Cohen DM, et al. Mechanical tugging force regulates the size of cell-cell junctions. Proc Natl Acad Sci USA 2010; 107:9944-9949.

31 Krajcovic M, Krishna S, Akkari L, Joyce JA, Overholtzer M. mTOR regulates phagosome and entotic vacuole fission. $\mathrm{Mol}$ Biol Cell 2013; 24:3736-3745.

32 Navin N, Kendall J, Troge J, et al. Tumour evolution inferred by single-cell sequencing. Nature 2011; 472:90-94.

33 Krajcovic M, Overholtzer M. Mechanisms of ploidy increase in human cancers: a new role for cell cannibalism. Cancer
Res 2012; 72:1596-1601.

34 Moreno E, Basler K, Morata G. Cells compete for decapentaplegic survival factor to prevent apoptosis in Drosophila wing development. Nature 2002; 416:755-759.

35 Lolo FN, Casas-Tinto S, Moreno E. Cell competition time line: winners kill losers, which are extruded and engulfed by hemocytes. Cell Rep 2012; 2:526-539.

36 Oliver ER, Saunders TL, Tarle SA, Glaser T. Ribosomal protein L24 defect in belly spot and tail (Bst), a mouse Minute. Development 2004; 131:3907-3920.

37 Sancho M, Di-Gregorio A, George N, et al. Competitive interactions eliminate unfit embryonic stem cells at the onset of differentiation. Dev Cell 2013; 26:19-30.

38 Claveria C, Giovinazzo G, Sierra R, Torres M. Myc-driven endogenous cell competition in the early mammalian embryo. Nature 2013; 500:39-44.

39 Marusyk A, Porter CC, Zaberezhnyy V, DeGregori J. Irradiation Selects for p53-Deficient Hematopoietic Progenitors. PLoS Biol 2010; 8:e1000324.

40 Bondar T, Medzhitov R. p53-mediated hematopoietic stem and progenitor cell competition. Cell Stem Cell 2010; 6:309322.

41 Shirasawa S, Furuse M, Yokoyama N, Sasazuki T. Altered growth of human colon cancer cell lines disrupted at activated Ki-ras. Science 1993; 260:85-88.

$42 \mathrm{Xu} \mathrm{X,} \mathrm{Omelchenko} \mathrm{T,} \mathrm{Hall} \mathrm{A.} \mathrm{LKB1} \mathrm{tumor} \mathrm{suppressor} \mathrm{pro-}$ tein regulates actin filament assembly through Rho and its exchange factor $\mathrm{Dbl}$ independently of kinase activity. $B M C$ Cell Biol 2010; 11:77.

43 Noren NK, Arthur WT, Burridge K. Cadherin engagement inhibits RhoA via p190RhoGAP. J Biol Chem 2003; 278:1361513618.

44 Sun Q, Overholtzer M. Methods for the study of entosis. Methods Mol Biol 2013; 1004:59-66.

45 Sun Q, Zhang Y, Yang G, et al. Transforming growth factor-beta-regulated miR-24 promotes skeletal muscle differentiation. Nucleic Acids Res 2008; 36:2690-2699.

46 Zhang Y, Fan KJ, Sun Q, et al. Functional screening for miRNAs targeting Smad4 identified miR-199a as a negative regulator of TGF-beta signalling pathway. Nucleic Acids Res 2012; 40:9286-9297.

47 Sun Q, Wang Y, Zhang Y, et al. Expression profiling reveals dysregulation of cellular cytoskeletal genes in $\mathrm{HBx}$-induced hepatocarcinogenesis. Cancer Biol Ther 2007; 6:668-674.

48 Zhou Q, Kee YS, Poirier CC, et al. 14-3-3 coordinates microtubules, Rac, and myosin II to control cell mechanics and cytokinesis. Curr Biol 2010; 20:1881-1889.

49 Hochmuth RM. Micropipette aspiration of living cells. J Biomech 2000; 33:15-22.

(Supplementary information is linked to the online version of the paper on the Cell Research website.) 